\title{
Understanding the structural evolution and stability of a Ge-Sn alloy at the nanoscale through in situ TEM heating
}

\author{
Alexey Minenkov and Heiko Groiss
}

Johannes Kepler University Linz / Center for Surface and Nanoanalytics, Linz, Oberosterreich, Austria

In a frame of ongoing miniaturization of semiconductor engineering, there is a great need for stable and efficient nanomaterials with advanced pre-desired properties. In this regard, the Ge-Sn alloys look exceedingly promising for the cutting-edge application in electronic and photonic devices due to their adjustable bandgap [1], compatibility with current Si-based technology, and one of the lowest contact resistivity among metal/ptype semiconductors. According to recent results, the Sn concentration in the alloy, which assures indirect-todirect bandgap transition should be more than 6.5 at.\% [1]. However, this value significantly exceeds the known bulk equilibrium solubility of $\mathrm{Sn}$ in $\mathrm{Ge}$, which makes the desired alloys drastically nonequilibrium [2]. Despite the considerable applied interest and promising properties of the crystalline Ge-based alloys with high Sn concentrations, the phases evolution, components interaction, and thermal stability at the nanoscale are still far from a full understanding.

We address this challenge with comprehensive in situ TEM investigation to monitor the interphase interactions in a nanoscale Ge-Sn film system during heating to the eutectic temperature and even beyond. We have used a series of thin, layered Ge-Sn films formed via sequential thermal evaporation (physical vapor deposition, PVD) as a convenient model of nano-binaries. The utilization of the thin amorphous (a)-Ge layer (in the case of PVD synthesis at room temperature) covered with Sn allowed us to follow the full metal-induced crystallization (MIC) of the a-Ge leading to the formation of highly uniform $\mathrm{Ge}_{1-\mathrm{x}} \mathrm{Sn}_{\mathrm{x}}$ alloy with a quite enhanced Sn content of 33 at.\%. We found that MIC temperature in the system under study could be as low as $90^{\circ} \mathrm{C}$ (Figure 1). We thoroughly traced on the fly the morphological and structural changes in the Ge-Sn system during heat-treatment in our JEOL JEM - 2200FS TEM equipped with MEMS-based Wildfire heating-holder from DENSsolutions. These results are well documented with video sequences [3]. Our observations of the crystal structure evolution convinced us that the MIC process at $90^{\circ} \mathrm{C}$ has been additionally facilitated by the formation of a metastable liquid phase at the metal-semiconductor interface. A combination of complementary microscopic techniques (HRTEM, HAADF STEM, SAED) in conjunction with plain-view and cross-sectional EDX elemental mapping allows us to discuss the evolution steps in the $\mathrm{Sn} / \mathrm{Ge}$ nanofilm on the way towards the $\mathrm{Ge}_{1-\mathrm{x}} \mathrm{Sn}_{\mathrm{x}}$ solid solution. We also traced the onset of its decomposition at elevated temperatures and highlighted the role of metastable and stable Sn-rich liquid phases in the thermal stability of the system (see Figure 2).

The presented findings could advance the fundamental understanding of the nanoobjects phase evolution during heat treatment and, in particular, empower new insight into components interactions in a Ge-Sn nanoscale system, which is of principal importance for controlled synthesis and application of these alloys. The data on the effect of size on investigated processes achievable alloy concentration, and its thermal stability as well as perspectives of the further development and implementation of the elaborated TEM techniques will be presented and discussed.

Acknowledgment: We are grateful for financial support from the Austrian Federal Ministry for Digital and Economic Affairs, the National Foundation for Research, Technology and Development and the Christian Doppler Research Association. 


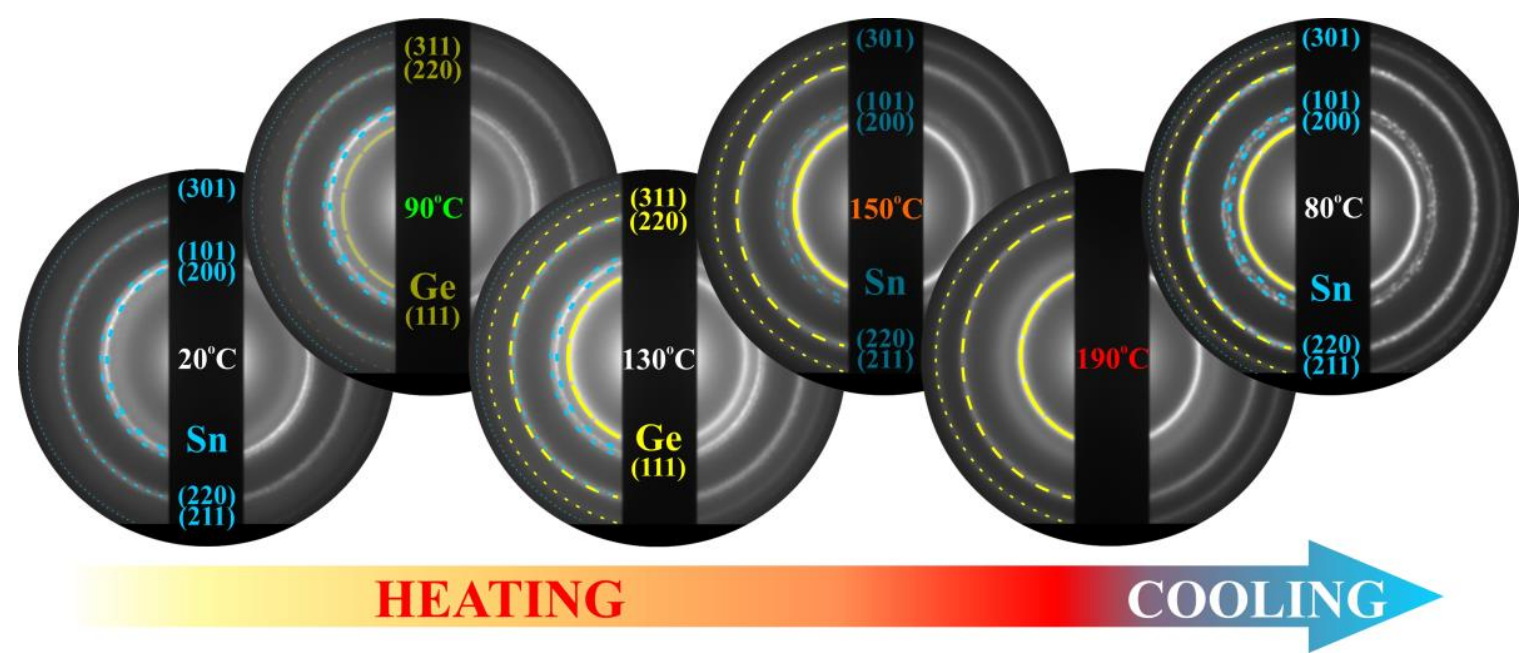

Figure 1. SAED patterns of the $\mathrm{Sn} / \mathrm{Ge}$ film snaped during a continuous heating-cooling cycle.

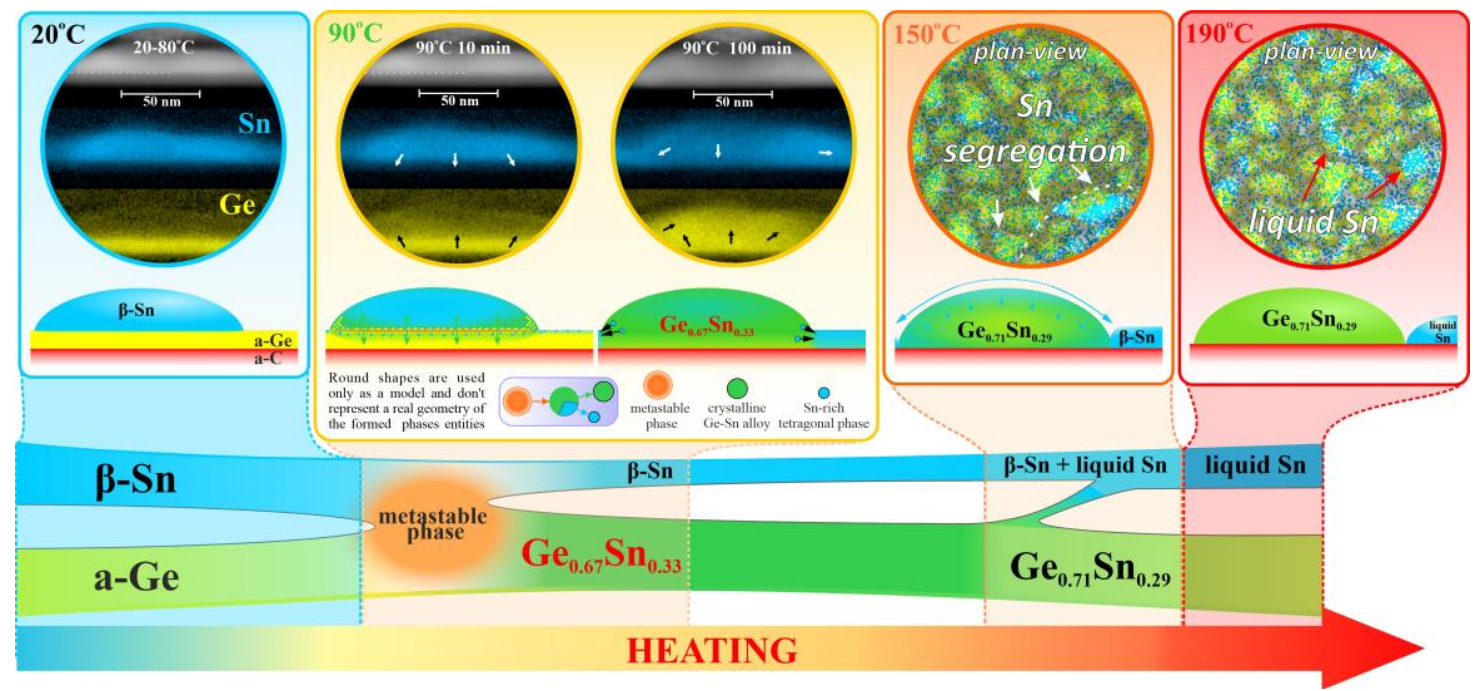

Figure 2. The scheme of the interphase interactions in $\mathrm{Sn} / \mathrm{Ge}$ film with the correspondent cross-sectional and plan-view images, accompanied by the diagram of the general phase's evolution in the sample during the heattreatment. Compilation of data presented in our recent article [3].

\section{References}

[1] Jessica Doherty et al., Progress on Germanium-Tin Nanoscale Alloys, Chem. Mater. 2020, 32, 11, 43834408.

[2] H. Groiss et al., Free-running Sn precipitates: An efficient phase separation mechanism for metastable $\mathrm{Ge}_{1-}$ ${ }_{x} \mathrm{Sn}_{\mathrm{x}}$ epilayers, Sci. Rep., 2017, 7, 16114.

[3] Alexey Minenkov, Heiko Groiss, Evolution of phases and their thermal stability in Ge-Sn nanofilms: a comprehensive in situ TEM investigation, Journal of Alloys and Compounds, 2021, 859, 157763. 